\title{
Epidemiology and Clinical Management of Traumatic Spine Injuries at a Major Government Hospital in Cambodia
}

\author{
Jee-Hye Choi ${ }^{1,2}$, Paul J. Park ${ }^{1,3}$, Vuthy Din ${ }^{1}$, Nang Sam ${ }^{1}$, Vycheth $I^{1}{ }^{1}$, Kee B. Park ${ }^{1,4}$ \\ ${ }^{1}$ Department of Neurosurgery, Preah Kossamak Hospital, Phnom Penh, Cambodia \\ ${ }^{2}$ Department of Global Health Initiatives, New York University School of Medicine, New York, NY, USA \\ ${ }^{3}$ Department of Physiology and Cell Biology, University of Nevada Reno School of Medicine, Reno, NV, USA \\ ${ }^{4}$ Department of Global Health and Social Medicine, Harvard Medical School, Boston, MA, USA
}

\section{Study Design: Cross sectional study.}

Purpose: To characterize the pattern of injury, describe the current clinical management, and determine the outcomes in traumatic spine injury (TSI) patients presenting to a major government hospital in Phnom Penh, Cambodia.

Overview of Literature: There is a paucity of literature on epidemiology or current clinical practices for TSIs in Cambodia. The findings from this study can thus serve as a valuable resource for future progress in treating TSIs in low-income countries.

Methods: This study was a cross-sectional study of TSI patients admitted to Preah Kossamak Hospital in Phnom Penh, Cambodia. Demographics, cause of spinal injury, spinal level of injury, surgical procedures and techniques, complications, and American Spinal Injury Association (ASIA) grades were recorded and analyzed.

Results: Eighty patients were admitted with TSI between October 2013 and June 2014. Falls from heights were the most common cause of TSI, followed by road traffic accidents. $78 \%$ of the admitted patients underwent at least one surgical procedure. Without intraoperative imaging, 4 patients $(6 \%)$ had wrong level surgery, and 1 patient $(2 \%)$ had misplacement of pedicle screws. Sacral decubitus ulcers were the most common non-surgically related complication. Antibiotics were administered to $>90 \%$ of patients. There were no in-hospital mortalities. Of the 60 spinal cord injury (SCI) patients, 32\% (19/60) showed improvement in their ASIA grade at the time of discharge, and 52\% (31/60) showed no change. At follow-up, 32\% (19/60) of SCI patients reported improvement, and 8\% $(5 / 60)$ reported no change. However, $36 \mathrm{SCl}$ patients (60\%) were lost to follow-up.

Conclusions: Despite technological limitations, outcomes of TSI patients in Cambodia appear favorable with evidence of clinical improvement and low mortality.

Keywords: Spinal injuries; Epidemiology; Neurosurgical procedures; Global health; Cambodia

\section{Introduction}

Traumatic spine injuries (TSIs) and spinal cord injuries
(SCIs) are serious global public health problems with significant financial and emotional burdens on the patients and their families [1-3]. Although the epidemiology

\footnotetext{
Received Mar 24, 2017; Accepted Apr 13, 2017

Corresponding author: Kee B. Park

Department of Global Health and Social Medicine, Harvard Medical School, 641 Huntington Ave, Boston, MA 02115, USA

Tel: +1-573-587-2468, Fax: +1-617-432-2565, E-mail: kee_park@mail.harvard.edu
} 
of SCIs has been described in several low-and-middleincome countries (LMICs) such as Pakistan, Nigeria, and Ethiopia, little is still known about the current state of clinical management of TSIs and SCIs in these regions of lower economic status [4-10].

In LMICs, the limited resources that clinicians work with present a formidable challenge for adhering to the latest practice guidelines developed in higher-income countries. Because of the discrepancies between these guidelines and available resources, there is a need for clinical data that reflects the socioeconomic conditions of a region so that practice guidelines can be tailored to its specific needs and capabilities. In this regard, there is a paucity of information regarding the epidemiology or the surgical techniques currently being utilized to treat TSI and SCI patients in Cambodia. In order to better understand these, we performed a cross-sectional study at Preah Kossamak Hospital, a major government hospital in Phnom Penh, Cambodia. The aims of this study were to: (1) characterize the epidemiology of TSI patients presenting to Preah Kossamak Hospital, (2) elucidate any pattern of TSIs in relation to causes of trauma, (3) describe the surgical management of TSIs, and (4) determine outcomes of the patients. In addition to our study aims, we believe that our study data can serve as baseline for future progress in the clinical management of TSI and SCI patients in LMICs.

\section{Materials and Methods}

\section{Institutional Review Board approval}

This study was approved by the Cambodian National Ethics Committee for Health Research, Protocol no. 350 NECHR.

\section{Data collection}

This study was conducted in the Department of Neurosurgery at Preah Kossamak Hospital, one of five hospitals with instrumented spine surgery capabilities in Phnom Penh, Cambodia. All trauma patients admitted between October 2013 and June 2014 with radiographically-confirmed injuries to the osseous discoligamentous complex and/or neural components of the spine, with or without neurological deficit, were included in the study. A customized template for the collection and storage of patient data was developed on FileMaker Pro 12.0v5, and the patient information was collected from paper charts, direct interviews with patients and/or their families, and follow-up phone calls. Information regarding the surgical procedure and complications were recorded on an on-going basis.

\section{Classification of injury and outcome}

To identify the most common sites of TSI, the anatomical levels of all injuries were identified. Injury levels were categorized as cervical (C1-C7), cervicothoracic (injuries involving both $\mathrm{C} 7$ and $\mathrm{T} 12$ ), thoracic (T1-T12), thoracolumbar (injuries involving both T12 and L1), lumbar (L1L5), lumbosacral (injuries involving both L5 and S1), or sacral (S1-S2). The degree of neurological deficit from SCI was defined according to the International Neurological Classification of Spinal Injury using the American Spinal Injury Association (ASIA) Impairment Scale, which was recorded at time of admission, discharge, and at follow-up [11]. Follow-up information was collected via phone calls 1-6 months post-discharge.

\section{Surgical procedures}

Posterior stabilization and fusion (PSF) of the thoracolumbar spine was achieved using stainless steel Luque rods and wire (Uma Surgicals, Mumbai, India) for sublaminar fixation or with titanium pedicle screw fixation sets donated by Medyssey Co. Ltd. (Uijeongbu, Korea) and Solco Biomedical (Pyeongtaek, Korea) [12]. PSF with pedicle screw fixation was performed in less severe injuries that only required three levels of fusion (one level above and one level below site of injury), whereas Luque rod fixation was used in more severe injuries that required at least five levels of fusion (two levels above and two levels below the level of injury). Retropharyngeal approach in combination with iliac crest bone graft and stainless steel plates (Uma Surgicals) was used for anterior cervical diskectomy and fusion $(\mathrm{ACDF})$ and corpectomy procedures in the cervical spine. PSF with interspinous wiring in the cervical spine was performed using stainless steel wires, and the spinous processes were cut for use as bone graft spacers in cervical laminoplasty procedures. Localization of spinal level during surgery was performed primarily using anatomical landmarks in correlation with preoperative imaging studies due to absence of any intraoperative imaging e.g., $\mathrm{C}$-arm and portable $\mathrm{X}$-rays machines. The attending spine 
surgeons included 3 Cambodian neurosurgeons and 1 American neurosurgeon.

\section{Statistical analysis}

The two-sided chi-squared test was used to determine association between two categorical variables. Significance was defined as $p \leq 0.05$. For some of the analyses, patients with unknown or missing information were excluded.

\section{Results}

\section{Demographics}

During the 9-month period, 80 patients with radiographically confirmed traumatic spine injury were admitted to Preah Kossamak Hospital. Of 80 TSI patients, 84\% were male $(n=67)$ and $16 \%$ were female $(n=13)$ with a male-tofemale ratio of 5.2:1. The median age of the patients was 32 years with a range of 14 to 70 years. Sixty TSI patients (75\%) had an associated SCI, and 2 TSI patients (3\%) had

Table 1. Summary of TSI patient characteristics (no=80)

\begin{tabular}{|c|c|}
\hline Characteristics & No. $(\%)$ \\
\hline \multicolumn{2}{|l|}{ Age group (yr) } \\
\hline $0-15$ & $1(1)$ \\
\hline $16-30$ & $33(41)$ \\
\hline $31-45$ & $27(34)$ \\
\hline $46-60$ & $13(16)$ \\
\hline $61-75$ & $6(8)$ \\
\hline Mean age (standard deviation) & $37(13)$ \\
\hline Median age (range) & $32(14-70)$ \\
\hline \multicolumn{2}{|l|}{ Sex } \\
\hline Male & $67(84)$ \\
\hline Female & $13(16)$ \\
\hline \multicolumn{2}{|l|}{ Head injury } \\
\hline Yes & $2(3)$ \\
\hline No & $78(97)$ \\
\hline \multicolumn{2}{|l|}{ Spinal cord injury } \\
\hline Yes & $60(75)$ \\
\hline No & $20(25)$ \\
\hline \multicolumn{2}{|l|}{ Spine surgery } \\
\hline Yes & $62(78)$ \\
\hline No & $18(22)$ \\
\hline
\end{tabular}

TSI, traumatic spine injury. a concurrent head injury (Table 1).

\section{Cause of injury}

The predominant cause of TSI was falls from heights, accounting for $53 \%$ of cases $(n=42)$. The most common places of falls were trees and houses, accounting for $45 \%$ $(n=19)$ and $24 \%(n=10)$ of all fall cases, respectively. Other elevated points of fall included cars $(n=3)$, ladders $(n=1)$, buildings $(n=1)$, and construction sites $(n=1)$. The sites of the fall were not known for 7 fall patients.

The second most common cause of traumatic spine injury was road traffic accidents (RTA), accounting for $29 \%$ of cases ( $n=23)$. Seventeen of these cases were motorcyclerelated, while 6 were car-related. Other causes of injury included impact by falling wood $(n=3)$, animal-related accidents $(n=2)$, heavy-lifting $(n=1)$, house collapse $(n=1)$, and machine-related accidents $(n=1)$. The cause of injury was unknown for 7 patients.

\section{Levels of spinal injury}

A total of 122 individual levels of spinal injury were recorded for 75 patients (Fig. 1). The precise level of injury was not available for analysis in 5 of our patients. Of 75 patients, $57 \%(n=43)$ had more than one spinal level of injury. Overall, the highest frequency of injury was at L1 $(24 \% ; n=29)$ followed by T12 (12\%; $n=15)$ and C5 (11\%; $\mathrm{n}=14)$. No injuries were observed at the atlanto-axial complex of $\mathrm{C} 1$ and C2. Seven patients (9\%) had thoracolumbar junction injuries involving both T12 and L1, whereas no patients suffered a cervicothoracic junction injury involving both C7 and T1. One patient (1\%) suffered a lumbosacral junction injury involving both L5 and S1.

Interestingly, we discovered that the predominant sites of injury differed between falls, motorcycle accidents, and car accidents (Table 2). For example, falls were most often associated with lumbar spine injuries (62\%; 26 of 42 fall cases), whereas motorcycle accidents were most often associated with cervical spine injuries (59\%; 10 of 17 motorcycle RTA cases). In car accidents, $33 \%$ of the patients $(n=2)$ suffered cervical spine injuries, $33 \%(n=2)$ suffered lumbar spine injuries, and another $33 \%(\mathrm{n}=2)$ had injuries to the thoracic spine or thoracolumbar junction. The differences in the overall pattern of injury sites among different modes of injury were statistically significant $(p=0.03)$. 


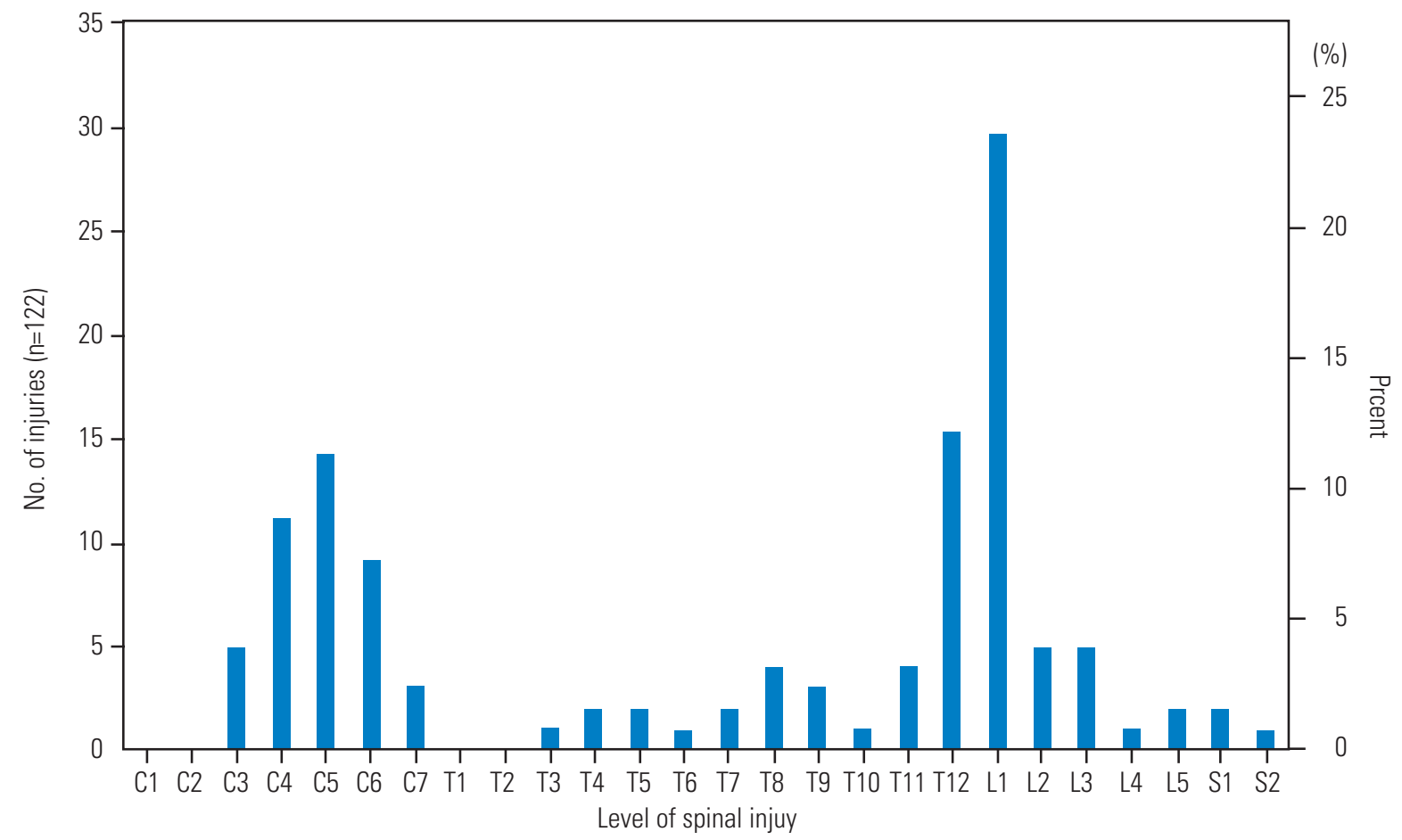

Fig. 1. Histogram analysis of all 122 spinal injury levels in 75 patients.

Table 2. Primary site of spinal injury associated with cause of injury in 80 patients

\begin{tabular}{|c|c|c|c|c|}
\hline Region of spinal injury & Falls & Motorcycle accidents & Car accidents & Other/unknown mechanisms \\
\hline Cervical & $6(14)$ & $10(59)$ & $2(33)$ & $4(27)$ \\
\hline Cervicothoracic & 0 & 0 & 0 & 0 \\
\hline Thoracic & $7(17)$ & $4(24)$ & $1(17)$ & $4(27)$ \\
\hline Thoracolumbar & $3(7)$ & 0 & $1(17)$ & $3(20)$ \\
\hline Lumbar & $26(62)$ & $2(12)$ & $2(33)$ & $3(20)$ \\
\hline Lumbosacral & 0 & $1(6)$ & 0 & 0 \\
\hline Sacral & 0 & 0 & 0 & $1(7)$ \\
\hline Total (no.) & $42(100)$ & $17(100)$ & $6(100)$ & $15(100)$ \\
\hline
\end{tabular}

Values are presented as number (\%).

\section{Presence of spinal cord injury (SCI) and ASIA grading}

The ASIA grading system was used to assess SCIs associated with TSIs upon admission (Table 3 ). More than onethird of TSI patients $(38 \% ; n=30)$ presented with a complete SCI (ASIA A), and an equal number of patients (38\%; $\mathrm{n}=30$ ) presented with an incomplete SCI (ASIA B, C, or D). One-fourth of TSI patients $(25 \% ; n=20)$ did not have any neurologic deficits upon presentation.
Table 3. ASIA grading and surgical management in 80 TSI patients

\begin{tabular}{lcc} 
Spinal cord injury & No. (\%) & Surgically treated \\
\hline Complete (ASIA A) & $30(38)$ & $25(83)$ \\
Incomplete (ASIA B, C, or D) & $30(38)$ & $21(70)$ \\
No SCl & $20(25)$ & $16(80)$ \\
Total (no.) & $80(100)$ & $62(78)$ \\
\hline
\end{tabular}

Values are presented as number (\%).

TSI, traumatic spine injury; ASIA, American Spinal Injury Association; $\mathrm{SCl}$, spinal cord injurie. 


\section{Antibiotics use}

Antibiotics records were obtained for 60 of the 80 TSI patients. Of those 60 patients, 54 patients (90\%) had documentation of receiving prophylactic antibiotics. Ceftriaxone, a third-generation cephalosporin, was used most frequently, followed by gentamicin, ciprofloxacin, levofloxacin, ampicillin, and cefpimizole.

\section{Surgical procedures}

Collectively, 78\% ( $n=62)$ of 80 TSI patients were managed surgically. The surgical rates for complete, incomplete, and no SCI were $83 \%(25 / 30), 70 \%(21 / 30)$, and $80 \%(16 / 20)$, respectively, and these differences were not statistically significant $(p=0.44)$.

Including revisions and complication treatments, there were a total of 75 surgical procedures for 62 patients (Table 4 ). The most common surgical procedure in our patient group was PSF with pedicle screw fixation $(40 \%$; 30/75) followed by PSF via Luque rod fixation with sublaminar wiring $(20 \% ; 15 / 75)$. One patient with a sacral zone II fracture involving S1 and S2 (1\%) was surgically treated with laminoforaminotomy and decompression of the symptomatic nerve root. All surgical procedures on the

Table 4. All spine surgical procedures performed on 62 TSI patients

\begin{tabular}{lc} 
Surgical procedure & No. (\%) \\
Thoracolumbar/sacral & $30(40)$ \\
\hline Posterior spinal fusion with pedicle screw fixation & $15(20)$ \\
\hline Posterior spinal fusion with Luque rod fixation & $1(1)$ \\
\hline Laminoforaminotomy & \\
\hline Cervical & $9(12)$ \\
\hline Anterior cervical diskectomy and fusion with plating & $5(7)$ \\
\hline Posterior spinal fusion with interspinous wiring & $5(7)$ \\
\hline Cervical laminoplasty & $1(1)$ \\
\hline Cervical corpectomy with bone graft & \\
\hline Revisions & $3(4)$ \\
\hline Revision of pedicle screw placement & $3(4)$ \\
\hline Revision of Luque rod placement & $1(1)$ \\
\hline Conversion from pedicle screw to Luque rod fixation & $1(1)$ \\
\hline Evacuation of postoperative hematoma & $1(1)$ \\
\hline Incision and drainage of pelvic abscess & $75(100)$ \\
\hline Total no. of surgical procedures for spinal injury (no.) & \\
\hline
\end{tabular}

TSI, traumatic spine injury. thoracic and lumbar spine and sacrum were posterior approaches, and the two types of PSF were used to surgically manage $78 \%(45 / 58)$ of all thoracic, lumbar, and sacral TSI.

The third most common procedure was ACDF with plating (12\%; 9/75), which accounted for $45 \%$ (9/20) of all cervical spine surgeries. Posterior approaches to the cervical spine included PSF with interspinous wiring (7\%; $5 / 75)$ and cervical laminoplasty (7\%; 5/75), which together accounted for $50 \%$ (10/20) of cervical spine procedures. In addition, 25\% (4/16) of cervical surgery patients underwent both an anterior $(\mathrm{ACDF}, \mathrm{n}=4)$ and a posterior procedure (cervical laminoplasty, $\mathrm{n}=3$; PSF with interspinous wiring, $n=1)$, whereas $38 \%(6 / 16)$ of cervical surgery patients had only an anterior procedure (ACDF, $n=5$; corpectomy with bone graft, $n=1)$. Furthermore, $38 \%(6 / 16)$ of cervical surgery patients underwent only a posterior procedure consisting of either PSF with interspinous wiring $(n=4)$ or cervical laminoplasty $(n=2)$.

Of the 62 TSI patients who had surgery, 7 patients (11\%) had suboptimal surgical results and required additional operations, including revision procedures (Table 4). One patient was converted from a PSF with pedicle screw fixation to Luque rod fixation with extension of the fusion levels because the postoperative spine stability achieved with the initial surgery was deemed inadequate. Overall, $15 \%(9 / 62)$ of surgical patients required a secondary operation.

\section{Complications}

Table 5. Complications of spine surgery in 62 surgical patients

Complication $\quad$ No. $(\%) \quad \begin{gathered}\% \text { Of } 62 \text { surgical } \\ \text { patients }\end{gathered}$

Intraoperative

$\begin{array}{lcl}\text { Wrong level surgery } & 4(27) & 6 \\ \text { Misplacement of pedicle screw } & 1(7) & 2 \\ \text { Incidental durotomy (cervical) } & 1(7) & 2\end{array}$

Postoperative

\begin{tabular}{lcc}
\hline Sacral decubitus ulcer & $5(33)$ & 8 \\
\hline Superficial wound infection & $1(7)$ & 2 \\
\hline Superficial wound dehiscence & $1(7)$ & 2 \\
\hline Pelvic abscess & $1(7)$ & 2 \\
Symptomatic hematoma & $1(7)$ & 2 \\
\hline Total (no.) & $15(100)$ & 24 \\
\hline
\end{tabular}


Table 5 shows that the overall complication rate for the 62 patients who underwent surgery was $24 \%(n=15)$. The most common intraoperative complication was wrong level surgery $(6 \% ; 4 / 62)$, which were all within the lumbar spine. The most common postoperative complication was sacral decubitus ulcer $(8 \% ; 5 / 62)$. There were no clinically evident cases of deep venous thrombosis, pulmonary embolism, myocardial infarction, intestinal obstruction, pneumonia, or sepsis. Although Foley urinary catheters were used, urine samples were not routinely tested or cultured to diagnose urinary tract infections.

\section{Outcome}

The length of hospital stay was recorded for 55 of 80 TSI patients (69\%). Their mean length of hospitalization was 18 days (standard deviation=15). The median was 16 days with a range of 0 to 108 days. Stratification into 5-day periods showed that the largest group of TSI patients (38\%; 21/55) stayed in the hospital between 16 to 20 days (Fig. 2).

Overall, no mortalities or worsening neurologic function was observed during hospitalization for all 80 TSI patients. Moreover, no mortalities were reported during 1-6-month follow-up phone calls for those patients who could be reached.

Initially, 60 of 80 TSI patients had shown signs of neurologic dysfunction at presentation (ASIA grades A-D). Of these 60 SCI patients, ASIA grade was recorded for $83 \%$ $(n=50)$ at the time of discharge. 38\% (19/50) of patients showed improved ASIA grades by the time of discharge,

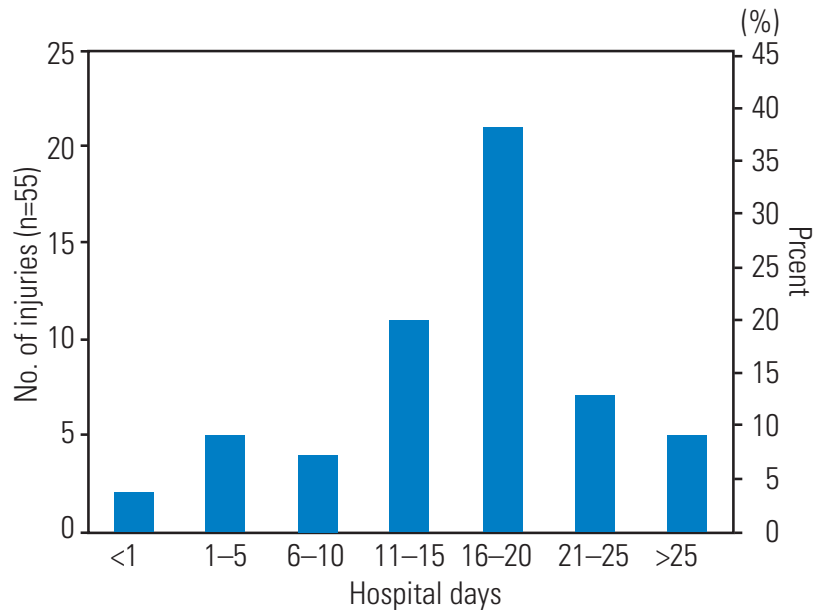

Fig. 2. Length of hospital stay in 55 TSI patients. Mean, 18 days; standard deviation, 15; median, 16; range, 0-108. TSI, traumatic spine injury. while $62 \%(31 / 50)$ patients had no change in their ASIA grades. Follow-up with telephone at 1-6 months after discharge revealed further improvements in neurologic function for some patients, with only one patient worsening after discharge. Unfortunately, a complete analysis was difficult due to a high number of patients who were lostto-follow-up (Table 6).

\section{Discussion}

We present here the first description of the epidemiology and the current clinical management of TSIs in Cambodia. Although our sampling is limited to 80 patients, the histogram analysis of thoracolumbar spinal injury levels in our patients resembles that of Magerl et al. [13], in which the thoracolumbar injury patterns of 1,445 patients were analyzed. Overall, the thoracolumbar junction was shown to be the most vulnerable site of injury in our analysis. Stratification by causes of injury showed that falls were most often associated with lumbar spine injuries, whereas motorcycle accidents were most often associated with cervical spine injuries. Within the cervical spine, C5 was the most vulnerable site of injury followed by $\mathrm{C} 4$ and C6. No injuries were observed at C1, C2, T1, and T2.

The SCI status (complete, incomplete, or no SCI) did not determine whether a patient was treated surgically or conservatively. The surgical treatment for complete SCI is controversial. Although many spine surgeons advocate early fusion of patients with complete SCI to enable maximal participation in physical rehabilitation, Shamim et al. [9] showed that in Pakistan, complete SCI patients who had surgery took longer time to go to rehabilitation, had higher rates of complications, and had longer hospital stays. Based on their findings, the authors recommended surgery for complete SCI patients only if their rehabilitation was impeded by pain or deformity. Continuing studies monitoring the clinical progression of SCI patients in rehabilitation programs who did and did not have surgery would reveal more accurate information about whether surgery should be performed readily on patients with complete SCI. At the of this study, we recognized that there was a paucity of inpatient rehabilitation programs in Cambodia, which would make such monitoring difficult.

Due to familiarity and simplicity of posterior approaches as well as lack of anterior instrumentation, all thoracolumbar cases were performed through a posterior approach. Although PSF with pedicle screw fixation was 
Table 6. Outcome based on ASIA grading scale in 80 TSI patients

\begin{tabular}{|c|c|c|c|c|}
\hline ASIA grade on admission & Discharge & No. $(\%)$ & Follow-up at 1-6 mo & No. $(\%)$ \\
\hline \multirow{6}{*}{$A($ no. $=30)$} & $A$ & $21(70)$ & $A$ & $5(17)$ \\
\hline & B & $5(17)$ & B & $3(10)$ \\
\hline & C & $1(3)$ & C & $4(13)$ \\
\hline & $D$ & $1(3)$ & $D$ & $1(3)$ \\
\hline & $E$ & 0 & $E$ & 0 \\
\hline & Unknown & $2(7)$ & Unknown & $17(57)$ \\
\hline \multirow[t]{6}{*}{$B($ no.=9) } & A & 0 & A & 0 \\
\hline & B & $4(44)$ & $\mathrm{B}$ & 0 \\
\hline & C & $4(44)$ & C & $1(11)$ \\
\hline & $D$ & 0 & $D$ & $1(11)$ \\
\hline & $E$ & 0 & $E$ & $2(22)$ \\
\hline & Unknown & $1(11)$ & Unknown & $5(56)$ \\
\hline \multirow[t]{6}{*}{$C($ no. $=12)$} & $A$ & 0 & A & 0 \\
\hline & B & 0 & $\mathrm{~B}$ & 0 \\
\hline & C & $2(17)$ & C & 0 \\
\hline & D & $5(42)$ & D & $1(8)$ \\
\hline & $E$ & 1 (8) & $E$ & $2(17)$ \\
\hline & Unknown & $4(33)$ & Unknown & $9(75)$ \\
\hline \multirow[t]{6}{*}{$D($ no. $=9)$} & A & 0 & A & 0 \\
\hline & B & 0 & B & 0 \\
\hline & C & 0 & C & 0 \\
\hline & $D$ & $4(44)$ & $D$ & 0 \\
\hline & $E$ & $2(22)$ & $E$ & $4(44)$ \\
\hline & Unknown & $3(33)$ & Unknown & $5(56)$ \\
\hline \multirow[t]{6}{*}{$E(n o .=20)$} & $A$ & 0 & $A$ & 0 \\
\hline & $B$ & 0 & B & 0 \\
\hline & C & 0 & C & 0 \\
\hline & $D$ & 0 & $D$ & 0 \\
\hline & $E$ & $16(80)$ & $E$ & $11(55)$ \\
\hline & Unknown & $4(20)$ & Unknown & $9(45)$ \\
\hline
\end{tabular}

ASIA, American Spinal Injury Association; TSI, traumatic spine injury.

the treatment of choice for most injuries, the availability of pedicle screw sets were limited and therefore were used judiciously. In more severe injuries that required 5 or more levels of fusion, the Luque rod fixation with sublaminar wiring was employed [12]. Luque rod instrumentation is an older PSF technique that has gone out of fashion in developed countries. However, because a Luque rod can be purchased for $\$ 15$ (USD) in Cambodia, it is an attractive and cost-effective means for treating severe spinal injuries. Its multipoint fixation gives the Luque system high rigidity and strength. However, operating times are longer, and the stainless steel Luque rod and sublaminar can make interpretation of MRI scans more difficult due to artifacts. In the cervical spine, PSF with interspinous wiring was also a highly cost-effective technique that was used since lateral mass fixation sets were not available. Rationale for choosing spinal implants in resource-limited setting has been described in more detail elsewhere [14].

Excluding sacral decubitus ulcers, the overall surgicallyrelated complication rate was $16 \%(10 / 62)$, of which wrong level surgery was the most common complication (6\%; 4/62). This finding emphasizes the value of a C-arm 
and intraoperative plain film X-rays in reducing wrong level surgeries. On a cultural note, while institutional policies in high-income countries tend to view wrong level surgeries as sentinel events, both the Cambodian neurosurgeons and the patients viewed the errors as an acceptable risk to the surgery, especially in light of the limited resources with which they worked $[15,16]$.

Although five sacral decubitus ulcers were recorded (all surgical patients), we believe that lower grade sacral decubitus ulcers were not documented. Therefore, the $8 \%$ sacral decubitus ulcer rate is likely an underestimate of its overall occurrence. In Cambodia, family members often stay with the patient during the hospitalization and perform much of the nursing care. Although some families provided water filled padding for the patients, the hospital beds were very firm, making SCI patients susceptible to developing pressure sores. Furthermore, physical rehabilitation was almost nonexistent. With this realization, efforts were directed at educating family members of SCI patients about prevention of sacral decubitus ulcers and importance of rehabilitation activities.

Interestingly, there were no clinically evident cases of deep venous thrombosis, pulmonary embolism, myocardial infarction, intestinal obstruction/ileus, pneumonia, or urinary tract infection during the hospitalization of our patients. However, subclinical forms of these complications cannot be ruled out since these conditions were not routinely screened. Furthermore, our infection rates were unexpectedly low (2\%), and it is uncertain whether the generous use of prophylactic antibiotics may have contributed to this phenomenon. As a cautionary measure, the nurses performed meticulous wound care with daily betadine washes until sutures were removed. Besides antibiotic use, the lack of central line use may have contributed to reducing infection and sepsis rates.

The average length of hospital stay (18 days) represented the usual time required for complete wound healing and suture removal, which were prerequisites for discharge. Although in-hospital mortality rates of $9.3 \%-17.5 \%$ have been reported among SCI patients in Pakistan and Nigeria mostly due to infectious or thromboembolic events, none of our patients died during hospitalization $[5,6,9]$. The reason for this is not clear. It is conceivable that the patients in our study were self-selected; given that only $10 \%$ of our patients were admitted within 4 hours of their injury, it is likely that patients with severe injury had died before reaching the hospital. A study in India, Lalwani et al. [17] found that $11.7 \%$ of the mortality among their TSI patients occurred on route or in less than 3 hours of admission to the hospital. Preadmission deaths may also explain why we did not observe any injuries at the atlantoaxial complex, which can compromise the brainstem leading to cardiorespiratory demise. Furthermore, given that $56 \%(45 / 80)$ of our patients were lost to follow-up, it is possible that some of our patients may have died following discharge; a study in Ethiopia with 23 month average follow-up revealed that $17 \%$ of patients who underwent surgery for spine fractures died, all after discharge [4]. Efforts to improve patient follow-up through family members may help clarify this concern in the future.

Despite the poor follow-up, the short-term as well as the mid-term outcomes (at 1-6 months) were encouraging. Improvements were seen in patients admitted with all grades of SCI even though the average delay to surgery was 5 days. Historically, the timing of decompressive surgery following TSI has been controversial with some reviews recommending consideration of early intervention 8 to 24 hours following acute TSI $[13,14,18]$.

\section{Conclusions}

Despite significant limitations in medical resources, spine surgery in Cambodia appears promising with reasonable complication rates, no in-hospital mortality, and trends of clinical improvement. The overall surgical rate for TSI patients was $78 \%$ with no significant difference between the surgical rates of complete, incomplete, and non-SCI patients. Prophylactic antibiotics were used generously, and it is uncertain if antibiotic resistance is becoming a problem. Establishment of physical rehabilitation programs is needed to maximize functional outcomes and minimize secondary complications, and efforts should be made to improve follow-up of TSI patients.

\section{Conflict of Interest}

No potential conflict of interest relevant to this article was reported.

\section{Acknowledgments}

This study was made possible through grants from the Korean American Medical Foundation and Korean American Spine Society. The authors would also like to thank 
Ms. Smey Hong for her translation services and assistance with data collection, and Dr. Jack Rock for serving as an advisor for the development of the database.

\section{References}

1. Chiu WT, Lin HC, Lam C, Chu SF, Chiang YH, Tsai SH. Review paper: epidemiology of traumatic spinal cord injury: comparisons between developed and developing countries. Asia Pac J Public Health 2010;22:9-18.

2. Fehlings MG, Perrin RG. The timing of surgical intervention in the treatment of spinal cord injury: a systematic review of recent clinical evidence. Spine (Phila Pa 1976) 2006;31:S28-35.

3. Ning GZ, Wu Q, Li YL, Feng SQ. Epidemiology of traumatic spinal cord injury in Asia: a systematic review. J Spinal Cord Med 2012;35:229-39.

4. Lehre MA, Eriksen LM, Tirsit A, et al. Outcome in patients undergoing surgery for spinal injury in an Ethiopian hospital. J Neurosurg Spine 2015;23:772-9.

5. Nwankwo OE, Uche EO. Epidemiological and treatment profiles of spinal cord injury in southeast Nigeria. Spinal Cord 2013;51:448-52.

6. Obalum DC, Giwa SO, Adekoya-Cole TO, Enweluzo GO. Profile of spinal injuries in Lagos, Nigeria. Spinal Cord 2009;47:134-7.

7. Okonkwo CA. Spinal cord injuries in Enugu, Nigeria: preventable accidents. Paraplegia 1988;26:12-8.

8. Ibrahim A, Lee KY, Kanoo LL, et al. Epidemiology of spinal cord injury in Hospital Kuala Lumpur. Spine (Phila Pa 1976) 2013;38:419-24.

9. Shamim MS, Ali SF, Enam SA. Non-operative management is superior to surgical stabilization in spine injury patients with complete neurological deficits: a perspective study from a developing world country, Pakistan. Surg Neurol Int 2011;2:166.

10. Uy S, Akashi H, Taki K, Ito K. Current problems in national hospitals of Phnom Penh: finance and health care. Nagoya J Med Sci 2007;69:71-9.

11. Kirshblum SC, Burns SP, Biering-Sorensen F, et al. International standards for neurological classification of spinal cord injury (revised 2011). J Spinal Cord Med 2011;34:535-46.

12. Luque ER, Cassis N, Ramirez-Wiella G. Segmental spinal instrumentation in the treatment of fractures of the thoracolumbar spine. Spine (Phila Pa 1976) 1982;7:312-7.

13. Magerl F, Aebi M, Gertzbein SD, Harms J, Nazarian S. A comprehensive classification of thoracic and lumbar injuries. Eur Spine J 1994;3:184-201.

14. Park KB, Iv V. Spinal implants in resource-limited settings: "Keep It Simple". World Neurosurg 2016;86:36-8.

15. Jhawar BS, Mitsis D, Duggal N. Wrong-sided and wrong-level neurosurgery: a national survey. J Neurosurg Spine 2007;7:467-72.

16. Mody MG, Nourbakhsh A, Stahl DL, Gibbs M, Alfawareh M, Garges KJ. The prevalence of wrong level surgery among spine surgeons. Spine (Phila Pa 1976) 2008;33:194-8.

17. Lalwani S, Singh V, Trikha V, et al. Mortality profile of patients with traumatic spinal injuries at a level I trauma care centre in India. Indian J Med Res 2014;140:40-5.

18. Vaccaro AR, Daugherty RJ, Sheehan TP, et al. Neurologic outcome of early versus late surgery for cervical spinal cord injury. Spine (Phila Pa 1976) 1997;22:2609-13. 